\title{
The shape of intratidal resistance-volume and compliance-volume curves in mechanical ventilation - an animal study
}

\author{
Buehler $S^{1}$, Schumann $S^{1}$, Lichtwarck-Aschoff ${ }^{2}$, Lozano $S^{1}$, Guttmann $J^{1}$ \\ ${ }^{1}$ Dept. of Anesthesiology, Division for Experimental Anesthesiology, University Medical Center Freiburg, Ger- \\ many \\ ${ }^{2}$ Department of Medical Sciences, Clinical Physiology, Uppsala University, Sweden.
}

sarah.buehler@uniklinik-freiburg.de

\begin{abstract}
In respiratory system mechanics, the shape of the intratidal pulmonary compliance-volume curve can be used to detect atelectasis or overdistension in the mechanically ventilated lung and thus to optimise the ventilator setting in terms of positive end-expiratory pressure $(P E E P)$ and tidal volume $\left(V_{T}\right)$. To this end, a set of shapecategories had been suggested. Furthermore, a characteristic behaviour of the intratidal resistance is expected as a consequence of alveolar recruitment and overdistension of the airways. We inspect the intratidal compliance and resistance profiles in mechanically ventilated pigs and suggest a classification into slope-categories for the resistance profile which could be used in combination with the compliance shape-categories to optimize PEEP and $V_{T}$.
\end{abstract}

Keywords: respiratory system mechanics; mechanical ventilation; intratidal resistance; intratidal compliance

\section{Introduction}

In the analysis of respiratory system mechanics during controlled mechanical ventilation an increasing focus is put on a dynamic approach as inspiratory recruitment and overdistension occurs within one single breath. It has been shown that the non-linear shape of the intratidal compliance-volume curve indicates indirectly if the lung is ventilated at high or low intrapulmonary volume $[1,2]$ and could therefore be used to adjust positive endexpiratory pressure (PEEP) and tidal volume $\left(\mathrm{V}_{\mathrm{T}}\right)$ as shown in a systematic animal study of intratidal compliance (C) at various PEEP-levels ([3]). To make this concept applicable, 6 shape categories have been proposed by [1] (Fig. 1): strong and weak increase indicating recruitment, strong and weak decrease indicating overdistension, a hybrid shape indicating recruitment and overdistension, constant $\mathrm{C}$ indicating no recruitment or overdistension.

Likewise, changes in the intratidal resistance $(\mathrm{R})$ are expected depending on the status of the lung. While the volume-dependency of $\mathrm{R}$ in ARDS patients has been shown by [4] no systematic study of changes in the course of the resistance-volume $(\mathrm{R}-\mathrm{V})$ curve with $\mathrm{V}_{\mathrm{T}}$ and PEEP has been carried out.

Here, we present a systematic study of intratidal compliance and resistance changes depending on PEEP as well as on $\mathrm{V}_{\mathrm{T}}$ in an ARDS animal model and introduce a new set of "slope categories" for the resistance.

\section{Methods}

15 pigs with a mean body mass of $25-32 \mathrm{~kg}$ were ventilated in volume-controlled mode at two different PEEP levels ( 0 and 12 mbar) and three different $\mathrm{V}_{\mathrm{T}}$ at each PEEP level $(8,12$ and $16 \mathrm{ml} / \mathrm{kg}$ bodyweight $)$ in ran-

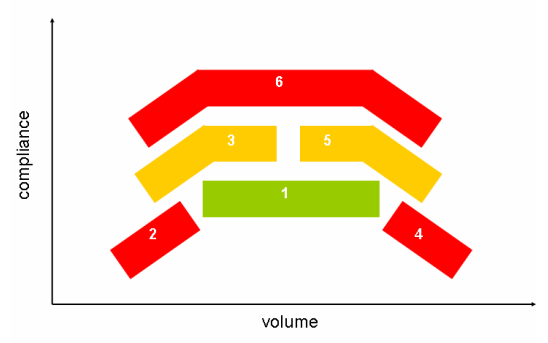

Figure 1: Schematic drawing of shape-categories compliance-volume curves.

dom order. Lung damage was induced by lavaging the pig lungs with saline solution. Measurements included continuous flow and pressure data as well as cardiac output and blood gas data.

Before assessing the intratidal course of R, C and the dynamic pressure base $\mathrm{P} 0$ with the gliding-SLICE method [3], we carried out expiratory low-pass filtering to eliminate disturbances from cardiogenic oscillations in the breathing signal. We setup our gliding-SLICE routine to divide the inner $10-90 \%$ of the pressure-volume loop into 91 overlapping slices and to solve the equation of motion of the respiratory system for each slice using a leastsquare algorithm. Nonlinearities from the endotracheal tube were avoided by converting airway pressures to tracheal pressures in advance [5].

$\mathrm{R}$ and $\mathrm{C}$ values were averaged over 10-20 breaths per animal and ventilator setting. The resulting compliancevolume $(\mathrm{C}-\mathrm{V})$ curves were then associated with one of the shape categories shown in Fig. 1. For each animal and PEEP level, the overall slope of the R-V curve was evaluated and absolute limits for the classification into slopecategories were set using the mean slope of all animals.

\section{Results}

The shape of the $\mathrm{C}-\mathrm{V}$ curve is remarkably similar for different animals (Fig. 2). While at PEEP zero the compliance was increasing for all $\mathrm{V}_{\mathrm{T}}$, at PEEP 12 


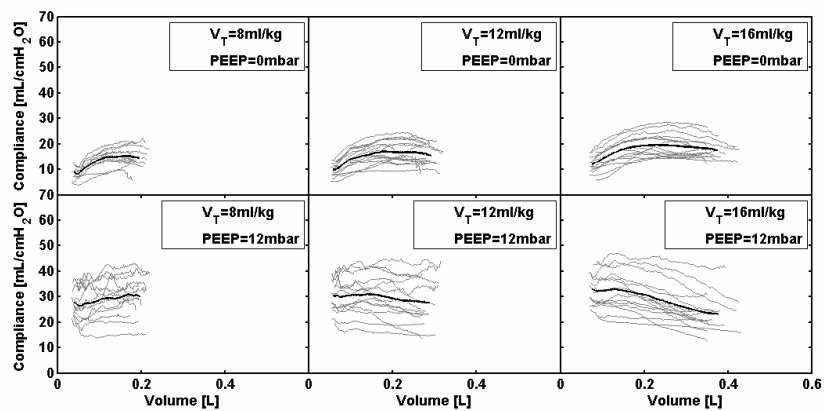

Figure 2: Compliance-volume curves at different PEEPlevels and tidal volumes. Each grey line represents the average over at least 10 breaths for every animal, the black lines show the average over all animals.

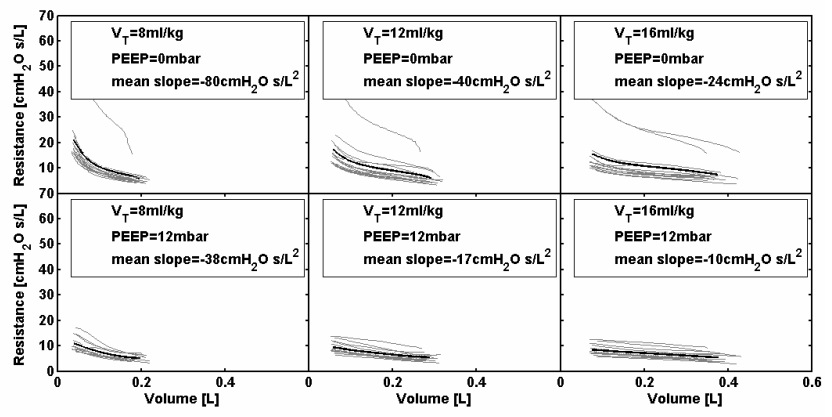

Figure 3: Same as Fig. 2 but for the resistance-volume curves. Mean slope values of all animals are given in the inserted boxes.

it remained constant at medium $\mathrm{V}_{\mathrm{T}}$ and decreased at large $\mathrm{V}_{\mathrm{T}}$.

Likewise, at each ventilation mode the shapes of the R-V curves of the individual animals were remarkably similar (Fig. 3). A large drop in $\mathrm{R}$ at small volumes and a slower decrease toward larger volumes can be seen. Furthermore, the total change in $\mathrm{R}$ over each breath decreased with increasing $V_{T}$ which resulted in a decreasing absolute slope with increasing $\mathrm{V}_{\mathrm{T}}$.

The absolute cuts for the resistance slope-categories were set at $-40 \mathrm{cmH}_{2} \mathrm{O} \mathrm{s} / \mathrm{L}^{2}$ (category 1), $-80 \mathrm{cmH}_{2} \mathrm{O} \mathrm{s} / \mathrm{L}^{2}$ (category 2) and $-24 \mathrm{cmH}_{2} \mathrm{O} \mathrm{s} / \mathrm{L}^{2}$ (category 3 ) for PEEP zero and at $-17 \mathrm{cmH}_{2} \mathrm{O} \mathrm{s} / \mathrm{L}^{2}$ (category 1), $-38 \mathrm{cmH}_{2} \mathrm{O} \mathrm{s} / \mathrm{L}^{2}$ (category 2) and $-10 \mathrm{cmH}_{2} \mathrm{O} \mathrm{s} / \mathrm{L}^{2}$ (category 3) for a PEEP of 12 mbar. A schematic drawing is shown in Fig. 4.

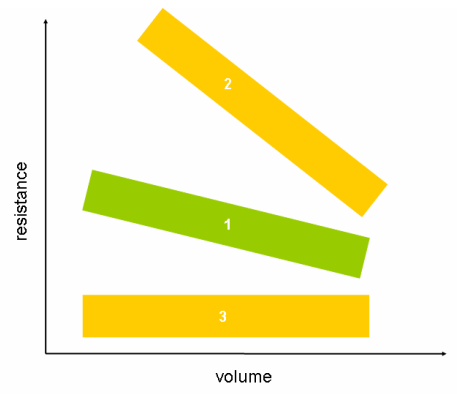

Figure 4: Schematic drawing of resistance slope-categories.

\section{Discussion}

We found a change in the shape of the compliancevolume curve depending on $\mathrm{V}_{\mathrm{T}}$. At PEEP zero it corresponded to a shape-category with increasing compliance (category 2 or 3 ) at all tidal volumes. An increasing compliance suggests intratidal recruitment which is in accordance with the collapsed lung we expect at PEEP zero. At PEEP 12 the shape-categories varied from increasing compliance (category 2 or 3 ) at low $\mathrm{V}_{\mathrm{T}}$ to constant compliance (category 1) at medium $\mathrm{V}_{\mathrm{T}}$ to decreasing compliance (category 4 or 5 ) at high $\mathrm{V}_{\mathrm{T}}$. This suggests intratidal recruitment at low $\mathrm{V}_{\mathrm{T}}$, overdistension at high $\mathrm{V}_{\mathrm{T}}$ and an optimum at medium $\mathrm{V}_{\mathrm{T}}$.

Furthermore, we found that the absolute slope of the resistance-volume curves decreased with increasing $\mathrm{V}_{\mathrm{T}}$ at both PEEP levels. It is hypothesized that a strong decrease at low tidal volumes is associated with the presence of atelectasis. The opening of the lung quickly leads to an increase in the airways' cross-sectional area and therefore to a decrease in $\mathrm{R}$ which is also reflected in the flattening at volumes around $0.15 \mathrm{l}$ - the less there is left to recruit the slower the decrease in R. A very small slope as seen at PEEP 12 and high $\mathrm{V}_{\mathrm{T}}$ or an even positive slope on the other hand might indicate overdistension as longitudinal stretching decreases the cross-section and slows down the decline of $\mathrm{R}$ [6]. An optimal ventilation setting is therefore argued to lie in between these slopes.

By classifying the intratidal course of the resistance into different slope-categories it might thus be possible to identify the optimal $\mathrm{V}_{\mathrm{T}}$ at a given PEEP-level. In combination with the compliance shape-categories this could provide a powerful tool for choosing a PEEP and $V_{T}$ setting which is in accordance with lung-protective ventilation.

\section{Bibliography}

[1] Mols, G. et al.: Volume-dependent compliance in ARDS: proposal of a new diagnostic concept, Intensive Care Med, vol. 25, pp. 1084-91., 1999.

[2] Mols, G. et al.: Alveolar recruitment in acute lung injury, Br J Anaesth, vol. 96, pp. 156-66, 2006.

[3] Schumann, S. et al.: Estimating intratidal nonlinearity of respiratory system mechanics: a model study using the enhanced gliding-SLICE method, Physiol Meas, vol. 30, pp. 1341-56, 2009.

[4] Mols, G. et al.: Is pulmonary resistance constant, within the range of tidal volume ventilation, in patients with ARDS?, Br J Anaesth, vol. 86, pp. 176-82., 2001.

[5] Guttmann, J. et al.: Continuous calculation of intratracheal pressure in tracheally intubated patients, Anesthesiology, vol. 79, pp. 503-13., 1993.

[6] Eissa, N. T. et al.: Effects of positive end-expiratory pressure, lung volume, and inspiratory flow on interrupter resistance in patients with adult respiratory distress syndrome, Am Rev Respir Dis, vol. 144, pp. 538-43, 1991. 